Cite this: Soft Matter, 2014, 10, 3978

\title{
Adding glycolipid functionality to model membranes - phase behaviour of a synthetic glycolipid in a phospholipid membrane $\uparrow$
}

\begin{abstract}
Urszula M. Migas, Lorna Abbey, Trinidad Velasco-Torrijos and Jennifer J. McManus*
Glycolipid phase behaviour is less well understood than for many phospholipids, but due to their structural and functional diversity, glycolipids represent an important group of amphiphiles from which biological function is derived. Here we have incorporated a synthetic glycolipid in binary mixtures with DOPC (1,2dioleoyl-sn-glycero-3-phosphocholine) into giant unilamellar vesicles (GUVs) at biologically relevant concentrations and observed the phase behaviour of the lipid mixtures for a range of glycolipid concentrations. At low concentrations, the glycolipid is fully dispersed in the GUV membrane. At glycolipid molar concentrations above $10 \%$, the formation of lipid tubules is observed, and is consistent with the formation of a columnar lipid phase. Lipid tubules are observed in aqueous and oil solvents, suggesting that both hexagonal and inverted hexagonal lipid arrangements can be formed. This work may offer insights into the biological function of glycolipids and the challenges in formulating them for use in industrial applications.
\end{abstract}

Received 19th January 2014

Accepted 1st April 2014

DOI: $10.1039 / c 4 s m 00147 h$

www.rsc.org/softmatter length of the carbon chain. The structure of the headgroup, mainly the configuration of the anomeric carbon as well as the number of sugar residues in the headgroup also influences the phase behaviour. ${ }^{19,20}$ When incorporated into vesicles, glycolipid molecules may be used to study the phase behaviour within model membranes. ${ }^{21,22}$

Due to the structural and functional diversity of naturally occurring and synthetic glycolipids, they are widely used in a range of academic and industrial applications. Glycolipid-based probes, containing a fluorophore or other type of tag, have been developed to study cell signalling and response, cellular uptake and trafficking. ${ }^{23}$

The specificity of carbohydrate interactions is of interest for targeted delivery. This is usually achieved by glycolipid-bearing liposomes containing for example, antigens necessary for immune system activation; ${ }^{24}$ a variety of drugs against inflammation $^{25}$ or as cancer therapies. ${ }^{26}$ The glycolipid headgroup provides the chemical moiety for targeted delivery. Sialyated Lewis antigens bind to selectin receptors present on the surface of endothelial cells, platelets and leukocytes and are widely studied due to their potential therapeutic application in facilitating delivery for inflammatory disease. ${ }^{27-29}$ There is also a growing interest in the role of glycolipids in bacterial and viral infection and therefore the potential for glycolipid-based therapeutic strategies..$^{30-33}$

Here we present experiments demonstrating a protected synthetic glycolipid, with a galactose-based headgroup and two asymmetrical hydrocarbon tails, has been successfully incorporated into phospholipid-cholesterol giant unilamellar vesicles (GUVs) at biologically relevant concentrations. The 
glycolipid-containing liposomes show good stability at room temperature. The synthetic glycolipid also shows interesting concentration-dependent phase behaviour in binary mixtures with DOPC and in ternary mixtures with DOPC and cholesterol, which will also be discussed. Here we use an acetylated synthetic glycolipid to demonstrate that these synthetically accessible lipid structures may be used as glycolipid mimics without the difficulties associated with purification of naturally occurring forms. The use of synthetic glycolipids offers the opportunity for further functionalization or modification after formation of self assembled structures, as demonstrated recently. ${ }^{34}$

\section{Experimental}

\section{Materials}

1,2-Dioleoyl-sn-glycero-3-phosphocholine (DOPC) and 1-myristoyl-2-[12-[(7-nitro-2-1,3-benzoxadiazol-4-yl)amino]-dodecanoyl]sn-glycero-3-phosphocholine (NBD-PC) (both highly purified) were purchased from Avanti Polar Lipids (Alabaster, Alabama). Cholesterol (highly purified) was purchased from Calbiochem, Merck (Darmstadt, Germany). Chloroform (analytical reagent grade) (Acros Organics), and Pt-metal wires (99.9\%) were purchased from Fisher Scientific (Ireland). Methanol (HPLC reagent grade), and mineral oil were purchased from Sigma Aldrich. Ethanol (HPLC reagent grade) was purchased from Lennox (Ireland). Imaging Spacer Secure-Seal ${ }^{\mathrm{TM}}$ and Perfusion Chamber Cover Well ${ }^{\mathrm{TM}}$ were purchased from GRACE Bio-Labs (Bend, Oregon). D-(+)-Saccharose was purchased from VWR International. $N$-(4,4-Difluoro-5,7-dimethyl-4-bora-3a,4a-diaza-sindacene-3-pentanoyl) sphingosine (bodipy $\mathrm{FL} \mathrm{C}_{5}$ ceramide), cholesteryl 4,4-difluoro-5,7-dimethyl-4-bora-3a,4a-diaza-s-indacene-3-dodecanoate (cholesteryl bodipy $\left.\mathrm{FL} \mathrm{C}_{12}\right), \quad \mathrm{N}-((4-(4,4-$ difluoro-5-(2-thienyl)-4-bora-3a,4a-diaza-s-indacene-3-yl) phenoxy) acetyl sphingosine (bodipy TR ceramide) (highly purified) were purchased from Invitrogen (Carlsbad, CA).

The synthesis of the glycolipid used in these experiments has been described previously. ${ }^{35}$

\section{GUV preparation}

W/O emulsion method (Method 1). A protocol based on the method of Noireaux and Libchaber ${ }^{36}$ was used. A lipid mixture of the desired lipid composition was dissolved in mineral oil at a concentration of $5 \mathrm{mg} \mathrm{ml}^{-1}$. The mixture was then heated and sonicated at $50{ }^{\circ} \mathrm{C}$ for 1 hour and incubated overnight at room temperature. $200 \mu \mathrm{l}$ of the clear supernatant and 1-1.5 $\mu \mathrm{l}$ of PBS buffer were vortexed for a few seconds to form a water-in-oil emulsion. After leaving the emulsion to rest for a few minutes, $50 \mu \mathrm{l}$ of the emulsion was placed on top of PBS buffer $(950 \mu \mathrm{l})$ and centrifuged at $2000 \mathrm{rpm}$ for 1 hour to form GUVs.

Hydration method (Method 2). A previously described method was used $;^{37} 20 \mu \mathrm{l}$ of lipid of the required composition, dissolved in chloroform $(0.1 \mathrm{M})$ was placed in a pear-shaped flask. $180 \mu \mathrm{l}$ of chloroform was added. Excess chloroform was removed by evaporation, forming a thin layer of lipid on the inner surface of the flask. The flask was then placed under vacuum for 24 hours to remove remaining chloroform. Nitrogen gas was passed through MilliQ water at $50{ }^{\circ} \mathrm{C}$ and then onto the dried lipid film for 40 minutes. Enough sucrose solution (0.1 M) was added to the flask to cover the lipid film. The flask was sealed and placed in an oven at $50{ }^{\circ} \mathrm{C}$ for 24 hours.

Rapid hydration method (Method 3). A protocol published by Moscho $^{38}$ was modified slightly as follows; $20 \mu \mathrm{l}$ of lipid of the required composition, dissolved in chloroform $(0.1 \mathrm{M})$ was placed in a pear-shaped flask. $150 \mu \mathrm{l}$ of ethanol and $900 \mu \mathrm{l}$ of chloroform were added. Milli-Q water $(7 \mathrm{ml})$ was added to the flask along the inside wall and the organic phase was removed using a rotary evaporator at temperature $c a .40{ }^{\circ} \mathrm{C}$.

\section{Imaging GUVs and size measurement}

Giant unilamellar vesicles were observed using an Olympus BX61 microscope, equipped with a digital imaging system, at either $60 \times$ or $100 \times$ magnification. Images of the structures formed were viewed and recorded using $\mathrm{Cell}^{\mathrm{F}}$ software. For fluorescence microscopy, a mercury lamp was used for excitation and images were viewed using band pass FITC or long pass Cy5 filter. All image analysis was done using Image ${ }^{39}$ and FigureJ software. ${ }^{40}$

\section{Results and discussion}

The phase behaviour of model membranes composed of a variety of lipid and cholesterol mixtures have been extensively studied. ${ }^{41-45}$ The presence of coexisting lamellar gel $\left(\mathrm{L}_{\beta}\right)$ and liquid-crystalline $\left(\mathrm{L}_{\alpha}\right)$ phases was previously shown for ternary lipid mixtures containing DOPC, cholesterol and DPPC or sphingomyelin (SM). In fact, only small differences were observed between phase diagrams of DOPC-cholesterol-DPPC and DOPC-cholesterol-SM. ${ }^{46,47}$ We have prepared 1:1:1 DOPC-cholesterol-SM lipid mixtures and have observed coexisting liquid phases (Fig. 1), as previously described. ${ }^{47}$

We are using a synthetic glycolipid molecule in which protecting groups are present instead of the $-\mathrm{OH}$ groups of a naturally occurring lipid. It has a flexible linker and two asymmetric hydrocarbon tails (Fig. 2). The synthesis for this glycolipid has been described previously. ${ }^{35}$ Our initial objective was

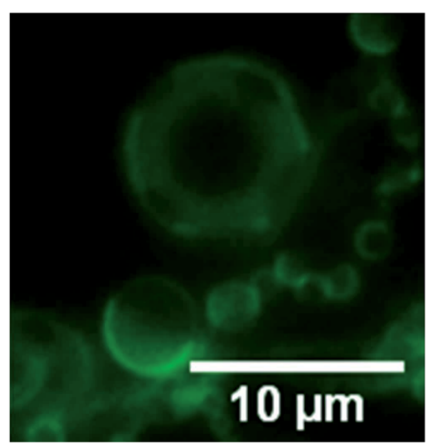

Fig. 1 Fluorescent microscopy images of GUVs containing $1: 1: 1$ DOPC-cholesterol-sphingomyelin (label is $0.05 \%$ cholesteryl bodipy $\mathrm{FL} \mathrm{C}_{12}$ ). 


\section{A}

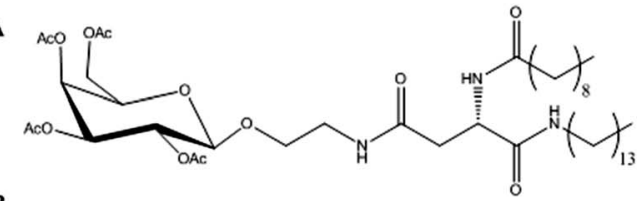<smiles>[Y]C(C)(C)C/C(C)=C/[C@H](O)[C@H](O)COP(=O)([O-])OCC[N+](C)(C)C</smiles>

C<smiles></smiles>

D<smiles></smiles>

E<smiles>CCCCCCCCC(=O)OC1CCC2(C)C(=CCC3C2CCC2(C)C3CCC2C(C)(C)CCC)C1</smiles>

Fig. 2 Chemical structures of the synthetic glycolipid (A), sphingomyelin (B), bodipy $F L C_{5}$ ceramide (C), bodipy TR ceramide (D) and cholesterol bodipy $\mathrm{FL}_{12}(\mathrm{E})$.

to show that it is possible to form GUVs containing this synthetic glycolipid, at biologically relevant concentrations and to investigate if liquid phase separation is observed within the membranes containing the protected form of the glycolipid.

A lipid film consisting of only the synthetic glycolipid (100\%) does not swell after hydration and therefore does not produce vesicles. The membranes we describe here contained binary mixtures of glycolipid (A) and DOPC or ternary mixtures of DOPC-glycolipid-cholesterol. The incorporation of glycolipid A into GUVs was possible using all of the methods we tried. In all experiments described here, a fluorescently labelled lipid was incorporated at a concentration of $0.05 \%, 0.1 \%$ or $1 \%$ (depending on the dye used).

In order to observe the membrane containing our synthetic glycolipid, we used either bodipy $\mathrm{FL}_{5}$ ceramide (C) or bodipy TR ceramide (D) (Fig. 2). In glycolipid-DOPC binary mixtures in the absence of cholesterol and with glycolipid concentrations from 1-5 mol\%, the glycolipid appears to be fully incorporated into the GUVs and no phase separation is observed. The presence of the labelled lipid in the vesicle is in itself not confirmation of the inclusion of the glycolipid in the GUV. However, the vesicles formed with the inclusion of glycolipid exhibit properties not present for vesicles formed in its absence. GUVs containing glycolipid tend to cluster into chains and groups
(Fig. 3). Narrower size distributions for vesicles containing glycolipid are also observed (Fig. S1 $\dagger$ ). After swelling of the lipid film, no residual material is observed, and thus we believe that up to $5 \%$ glycolipid, the synthetic lipid is fully incorporated into the GUVs.

The vesicles formed are in high yield and rather stable. We observed no changes in the shape or stability of the vesicles for up to 12 days (stored at room temperature) which is longer than for vesicles formed without the synthetic glycolipid. This may be due to hydrogen bonding interactions, which alter the polarity of the lipid headgroup as suggested recently. ${ }^{48}$ This increase in stability led to a decrease in the CVC (critical vesicle concentration) of a fatty acid membrane containing a glycolipid. ${ }^{48}$

As the concentration of glycolipid is increased from 5 to $10 \%$, we observe the formation of tubular structures with increasing abundance as the concentration of glycolipid increases (Fig. 4). At glycolipid concentrations from $10-30 \%$, the tubular structures co-exist with GUVs. It appears that the $90: 10$ DOPC-glycolipid composition at which the tubular structures appear, represents the maximum solubility of the synthetic glycolipid within the phospholipid in the lamellar phase. We observed the formation of the tubular structures in the presence and absence of fluorescent dyes, which eliminates the dyes as a reason for tubule formation (Fig. $\mathrm{S} 2 \dagger$ ).

To test if our chosen fluorescent label (bodipy $\mathrm{FL} \mathrm{C}_{5}$ ceramide) was possibly masking any phase separation within the lipid lamellar phase, we used a second dye, cholesteryl bodipy $\mathrm{FL} \mathrm{C}_{12}$, which partitions into the liquid ordered phase $\left(\mathrm{L}_{\mathrm{o}}\right)$, often called a lipid 'raft', which would allow us to observe liquid phase separation if the bodipy ceramide dyes failed to do so. ${ }^{49}$ In both cases, no liquid phase separation was observed. We do not expect DOPC or DOPC-cholesterol to form these types of structures in the absence of the glycolipid..$^{\mathbf{5 0} 51}$ To be absolutely

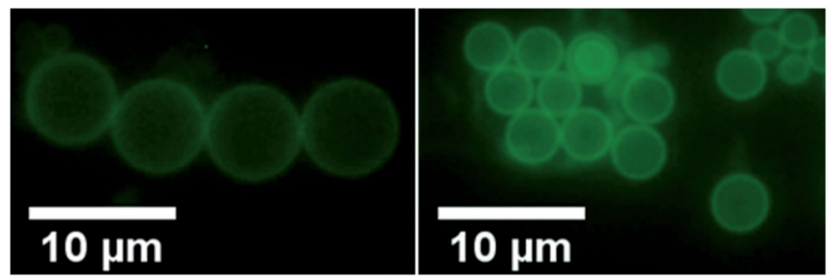

Fig. 3 Clusters of GUVs containing glycolipid formed by Method 2, composed of 1 : 9 glycolipid-phospholipid mixtures.
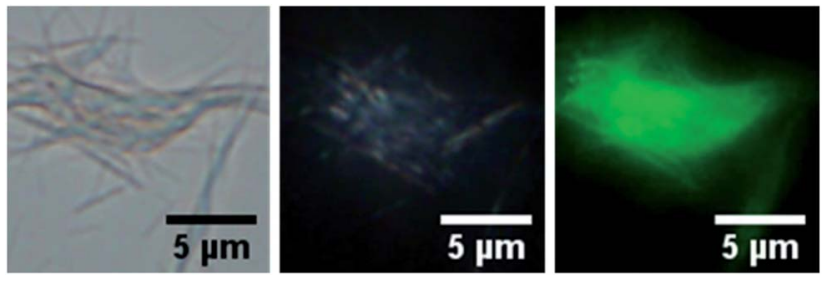

Fig. 4 Optical microscopy images of tubular structures observed in binary mixtures of DOPC-glycolipid. Left panel; phase contrast, middle panel; polarization; right panel, fluorescence due to labelled glycolipid. 
certain that liquid phase separation does not occur, we performed two-dye experiments (Fig. 5).

We prepared GUVs using an initial glycolipid concentration of $33 \mathrm{~mol} \%$, which is above the solubility of the glycolipid within the lamellar phase to ensure oversaturation. In these experiments, the glycolipid dye (bodipy $\mathrm{FL} \mathrm{C}_{5}$ ceramide or bodipy TR ceramide) was used with either cholesteryl bodipy FL or NBD PC. ${ }^{52}$ That is, we created a glycolipid-cholesterol twodye pair and a glycolipid-phospholipid two-dye pair to ensure that phase separation of the glycolipid from either the phospholipid or cholesterol was not occurring. No phase separation was observed in either case.

We also investigated if cholesterol could contribute to the formation of a liquid ordered, $\mathrm{L}_{\mathrm{o}}$ phase in the glycolipid containing membranes. We formed membranes with a fixed glycolipid concentration of $5 \%$ and varied the DOPC-cholesterol ratio so that the concentration of cholesterol varied from $10-30 \%$. With increasing cholesterol content, there is a lower yield of vesicles formed and a decrease in their size, most apparent at $30 \%$ cholesterol (Fig. S3†). However we did not observe coexistence of liquid-ordered and liquid-crystalline phases in the bilayers formed over the range of cholesterol concentrations used.

To ensure that the tubules formed are not artefacts of the preparation method selected, we prepared vesicles in a number of ways (Methods 1-3, Experimental). Methods 2 and 3 produce the same self-assembled structures at the same glycolipid concentrations (both GUVs and tubules at glycolipid concentrations above $10 \%$ ).

The emulsion based method (Method 1) shows slightly different behaviour. GUVs containing glycolipid are observed in the aqueous phase. Interestingly however the tubular structures that are observed in the aqueous phase for the hydration methods (Fig. 6A) are found inside the mineral oil droplets that occur in the emulsion method (Fig. 6B). The lipid tubules are optically birefringent indicating that the structures are ordered and not just aggregated material (Fig. 4 (middle panel)).
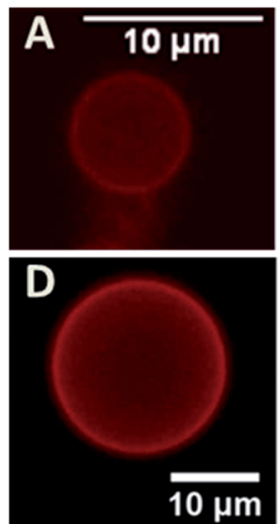
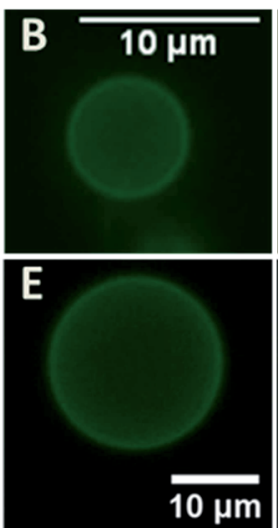
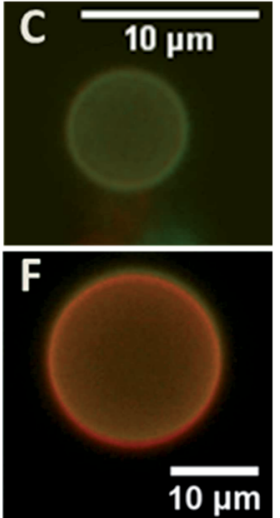

Fig. 5 Fluorescent microscopy images of GUVs $(1: 1: 1$ DOPCglycolipid-cholesterol) prepared by Method 2. (A-C) Labelled with $0.05 \%$ cholesteryl bodipy FL C12 and $0.05 \%$ bodipy TR ceramide; (D-F) labelled with $0.05 \%$ bodipy TR ceramide $1 \%$ NBD-PC; (C \& F) overlay of the two images.
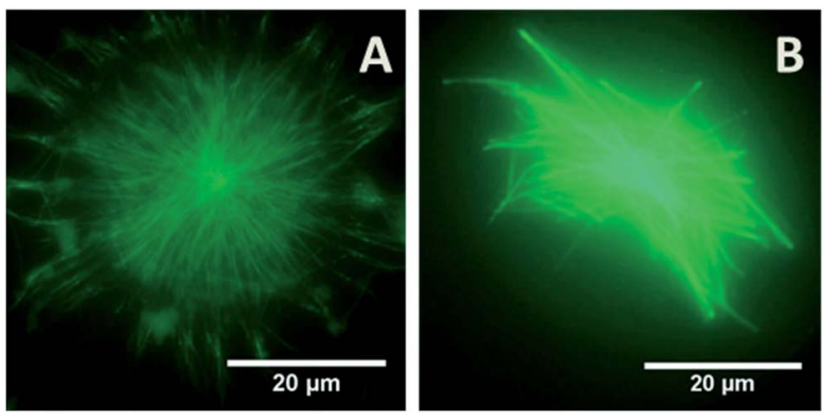

Fig. 6 Fluorescent microscopy images of tubules formed in aqueous solvent (A) and in mineral oil (B); lipid compositions are: $1: 9(\mathrm{~A})$ and $1: 4$ (B) glycolipid-DOPC labelled with $0.1 \%$ bodipy FL $\mathrm{C}_{5}$ ceramide.

Taken together, this behaviour is consistent with the formation of a columnar phase with hexagonally packed lipid tubules in the aqueous solvent and inverted hexagonally packed tubules in the mineral oil droplets. Given the sizes of the tubular features we see, each tubular structure would represent several layers of hexagonally arranged lipid tubes.

The formation of a non-lamellar phase is driven by a curvature stress on the membrane which is induced in the presence of the glycolipid. The tendency of lipid membranes to arrange themselves in specific phases may be qualitatively described by the Israelachvili-Mitchell-Ninham packing parameter, $P=v / a l$, where $v$ is the molecular volume, $a$ is the cross-sectional area of the headgroup and $l$ is the length of the molecule. ${ }^{53}$ We presume that up to a molar concentration of $5 \%$, the glycolipid is fully dispersed within the DOPC lamellar phase in the GUVs as indicated earlier. We only observe tubular structures at molar concentrations of $10 \%$, which suggests that if the glycolipid is dispersed equally between the lamellar and hexagonal phases that a minimum of $5 \%$ glycolipid is required for the formation of a hexagonal phase. In reality, we do not know if there is an equal distribution of glycolipid between the two phases. Lamellar phases are favoured at $P$ values close to $1 .^{53}$ The glycolipid has both a larger cross-sectional area of the headgroup, $a$, due to the bulky sugar and a smaller molecular volume, $v$, than DOPC in the aqueous phase due to its asymmetric tails. With increasing glycolipid concentrations this will result in a value of $P<1$ and hence the formation of a hexagonal phase. The value of $P$ should exceed 1 for the formation of an inverted hexagonal phase. The inverted hexagonal phase forms in a mineral oil solvent for this system, hence the values for $a, v$ and $l$ for the same lipid will change due to the different solvent environment. In this system, a reorientation of the lipid headgroup towards the lipid tails, is plausible due to the flexible linker and would result in a decrease in the values of both $a$ and $l$ and lead to a packing parameter, $P>1$.

Both $\mathrm{H}_{\mathrm{I}}$ and $\mathrm{H}_{\mathrm{II}}$ phases have been observed for glycolipids previously. ${ }^{17,54,55}$ Hence, there appears to be a transition from a $\mathrm{L}_{\alpha}$ lipid phase in the GUVs to a columnar $\mathrm{H}_{\mathrm{I}}$ or $\mathrm{H}_{\mathrm{II}}$ lipid phase at a glycolipid concentrations between 5 and 10\%, depending on whether the tubules are in an aqueous or mineral oil solvent. The proposed arrangement of the columnar phase is illustrated in Fig. 7. 

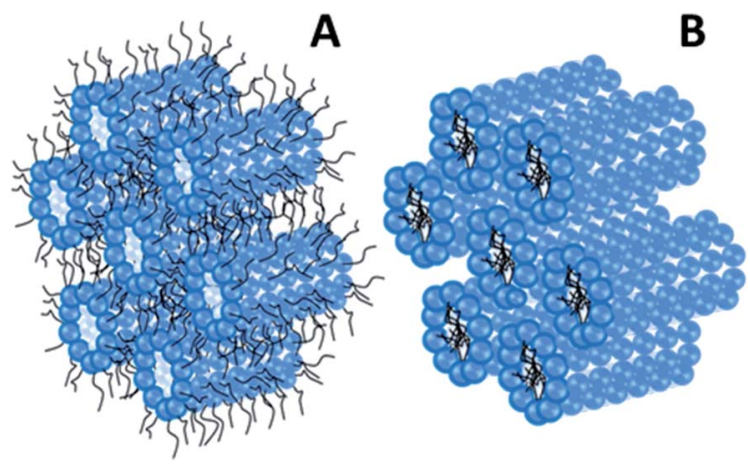

Fig. 7 Arrangement of lipids in columnar phases; (A) inverted hexagonal $\left(\mathrm{H}_{\| 1}\right)$ arrangement of lipids in which the glycolipid tail groups are oriented towards the mineral oil solvent and $(\mathrm{B})$ hexagonal $\left(\mathrm{H}_{1}\right)$ lipid packing, where the glycolipid headgroup is oriented towards the aqueous solvent. We believe the lipid layers are composed of glycolipid-phospholipid mixtures.

Given that our synthetic glycolipid has a slightly hydrophobic headgroup, we have considered the possibility that a $\mathrm{H}_{\mathrm{II}}$ phase forms, even in aqueous solvent. However, the hydrocarbon tails are sufficiently long, that a $\mathrm{H}_{\text {II }}$ phase in aqueous solvent would be entropically unfavourable and a $\mathrm{H}_{\mathrm{I}}$ phase is more likely. Further work will be required to confirm both the proposed structures and specific lipid arrangements in both aqueous and oil solvents.

We have confirmed that for the hydration methods used to form the GUVs that the lipid is fully dispersed with the DOPC and cholesterol in chloroform before vesicle formation and that the structures observed are not due to insolubility. We believe that the columnar phase is composed of both glycolipid and phospholipid, since the glycolipid does not by itself swell into an aqueous phase.

Since the fluorescent images show uniform distribution of the fluorescent dye, we assume that the lipid composition in the
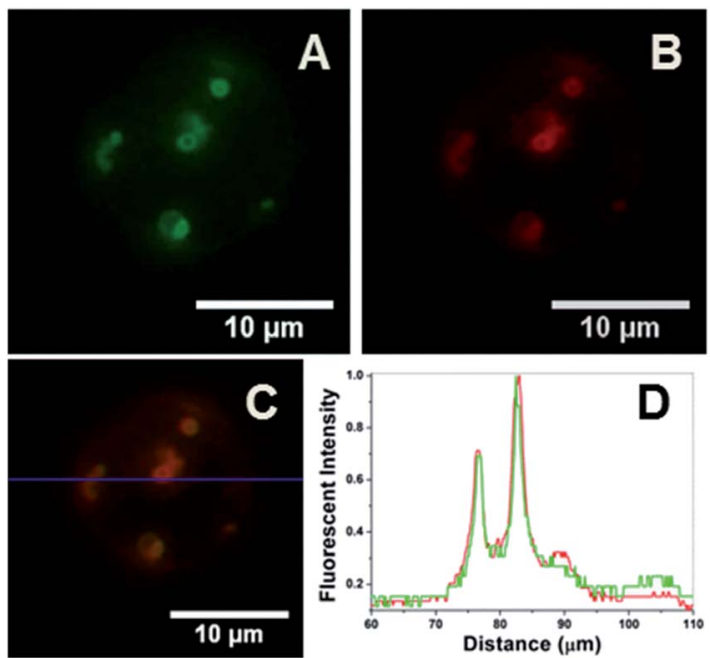

Fig. 8 Fluorescent images of GUVs attached to the surface of the mineral oil droplet; (A-C) $80: 20$ DOPC-glycolipid (0.1\% bodipy FL $C_{5}$ ceramide 1\% NBD-PC); (D) fluorescent intensity profile (cross-section intensity). tubules is homogeneous. When GUVs are formed by Method 1, several of them are found on the surface of mineral oil droplets. In order to analyse the lipid distribution on the surface of the mineral oil and to ensure that the glycolipid does not partition preferentially into the mineral oil solvent we performed fluorescent microscopy with two fluorescent dyes, bodipy TR ceramide and NBD-PC, known to partition into a phospholipid rich phase. Our choice of $20 \%$ glycolipid content was again dictated by the need to saturate the mineral oil, used in the first step of the preparation method.

As shown in Fig. 8 the two fluorescent lipids are localized in the same areas on the surface of the oil droplet and no phase separation of the synthetic glycolipid is induced on the surface of the mineral oil. This may also provide further evidence that both the glycolipid and phospholipid are required for tubule formation, since the vesicles on the mineral oil droplets are the likely source of material for the columnar phase to form.

\section{Conclusions}

A protected synthetic glycolipid has been incorporated into GUV membranes by a variety of methods at biologically relevant concentrations in binary mixtures with DOPC and in ternary mixtures with DOPC and cholesterol. In both cases, the glycolipid is fully dispersed in the lamellar phase and no liquid phase separation was observed in the range of concentrations where the glycolipid was soluble in the vesicle membrane. At glycolipid concentrations above $10 \%$, the formation of lipid tubules occurs, which we believe is most likely a hexagonally packed columnar lipid phase. The tubules are optically birefringent and occur both in aqueous solvent and in mineral oil droplets formed during GUV preparation by an emulsion based method. The study of the phase behaviour of both naturally occurring and synthetic glycolipids is important in understanding their role in biological processes. The effect of total lipid concentration on the aggregate structure has not been examined, but will be important to investigate in future work. The phase behaviour of glycolipids in mixtures with other membrane forming lipids has yet to be fully explored and this work is a contribution to this effort.

\section{Acknowledgements}

U.M.M. and J.J.McM acknowledge funding from the HEA under PRTLI 5, co-funded by the Irish Government and the EU under Ireland's Structural Funds Programmes 2007-2013: investing in your future. J.J.McM acknowledges funding from the SFI Stokes Lectureship. T.V.T. and L.A. acknowledge funding from the Irish Research Council.

\section{References}

1 K. Kasahara and Y. Sanai, Glycoconjugate J., 2000, 17, 153162.

2 A. R. Todeschini and S. I. Hakomori, Biochim. Biophys. Acta, 2008, 1780, 421-433.

3 R. Malhotra, Biochem. Anal. Biochem., 2012, 1, 108. 
4 M. A. Chester, IUPAC-IUB Joint Commission on Biochemical Nomenclature (JCBN), Glycoconjugate J., 1999, 16, 1-6.

5 M. Corti, L. Cantù, P. Brocca and E. Del Favero, Curr. Opin. Colloid Interface Sci., 2007, 12, 148-154.

6 M. W. Tate, E. F. Eikenberry, D. C. Turner, E. Shyamsunder and S. M. Gruner, Chem. Phys. Lipids, 1991, 57, 147-164.

7 I. Koltover, T. Salditt, J. O. Rädler and C. R. Safinya, Science, 1998, 281, 78-81.

8 S. Tristram-Nagle and J. F. Nagle, Chem. Phys. Lipids, 2004, 127, 3-14.

9 A. Bilalov, U. Olsson and B. Lindman, Soft Matter, 2012, 8, 11022-11033.

10 W. Shinoda, D. E. Discher, M. L. Klein and S. M. Loverde, Soft Matter, 2014, 9, 11549-11556.

11 A. R. Wellburn, New Phytol., 1997, 135, 115-121.

12 D. K. Hincha, I. Bakaltcheva and J. M. Schmitt, Plant Physiol., 1993, 103, 59-65.

13 G. Hölzl and P. Dörmann, Prog. Lipid Res., 2007, 46, 225-243.

14 A. Minoda, N. Sato, H. Nozaki, K. Okada, H. Takahashi, K. Sonoike and M. Tsuzuki, Eur. J. Biochem., 2002, 269, 2353-2358.

15 K. Nakata, C. T. Guo, M. Matsufuji, A. Yoshimoto, M. Inagaki, R. Higuchi and Y. Suzuki, J. Biochem., 2000, 127, 191-198.

16 B. M. Gadella, D. Hammache, G. Piéroni, B. Colenbrander, L. M. G. van Golde and J. Fantini, J. Reprod. Immunol., 1998, 41, 233-253.

17 J. M. Seddon, O. Ces, R. H. Templer, D. A. Mannock and R. N. McElhaney, Mol. Cryst. Liq. Cryst., 2003, 402, 77-84.

18 D. Marsh, Chem. Phys. Lipids, 2012, 165, 23-31.

19 D. A. Mannock and R. N. McElhaney, Curr. Opin. Colloid Interface Sci., 2004, 8, 426-447.

20 M. Hato, Curr. Opin. Colloid Interface Sci., 2001, 6, 268276.

21 V. Castro, S. V. Dvinskikh, G. Widmalm, D. Sandström and A. Maliniak, Biochim. Biophys. Acta, 2007, 1768, 24322437.

22 J. Kapla, B. Stevensson, M. Dahlberg and A. Maliniak, J. Phys. Chem. B, 2012, 116, 244-252.

23 B. L. Stocker and M. S. M. Timmer, ChemBioChem, 2013, 14, 1164-1184.

24 W. C. Chen, N. Kawasaki, C. M. Nycholat, S. Han, J. Pilotte, P. R. Crocker and J. C. Paulson, PLoS One, 2012, 7, 6.

25 N. Hashida, N. Ohguro, N. Yamazaki, Y. Arakawa, E. Oiki, H. Mashimo, N. Kurokawa and Y. Tano, Exp. Eye Res., 2008, 86, 138-149.

26 W. C. Chen, G. C. Completo, D. S. Sigal, P. R. Crocker, A. Saven and J. C. Paulson, Blood, 2010, 115, 4778-4786.

27 R. E. Bruehl, F. Dasgupta, T. R. Katsumoto, J. H. Tan, C. R. Bertozzi, W. Spevak, D. J. Ahn, S. D. Rosen and J. O. Nagy, Biochemistry, 2001, 40, 5964-5974.

28 C. S. Shi, G. Y. Shi, H. M. Hsiao, Y. C. Kao, K. L. Kuo, C. Y. Ma, C. H. Kuo, B. I. Chang, C. F. Chang, C. H. Lin, C. H. Wong and H. L. Wu, Blood, 2008, 112, 3661-3670.

29 W. L. Lin, C. F. Chang, C. S. Shi, G. Y. Shi and H. L. Wu, Arterioscler., Thromb., Vasc. Biol., 2013, 33, 2366-2373.
30 K. Venken, T. Decruy, S. Aspeslagh, S. Van Calengergh, B. N. Lambrecht and D. Elewaut, J. Immunol., 2013, 191, 2174-2183.

31 W. B. Puryear and S. Gummuluru, Adv. Exp. Med. Biol., 2013, 762, 131-153.

32 M. Iwamori, M. Nakasa, K. Yamazaki, Y. Iwamori, K. Tanaka, D. Aoki, S. Adachi and T. Nomura, Glycoconjugate J., 2012, 29, 199-209.

33 H. Ewers, W. Römer, A. E. Smith, K. Bacia, S. Dmitrieff, W. Chai, R. Mancini, J. Kartenbeck, V. Chambon, L. Berland, A. Oppenheim, G. Schwarzmann, T. Feizi, P. Schwille, P. Sens, A. Helenius and L. Johannes, Nat. Cell Biol., 2010, 12, 11-18.

34 U. Kauscher, M. C. A. Stuart, P. Drücker, H. J. Galla and B. J. Ravoo, Langmuir, 2013, 29, 7377-7383.

35 T. Velasco-Torrijos, L. Abbey and R. O'Flaherty, Molecules, 2012, 17, 11346-11362.

36 V. Noireaux and A. Libchaber, Proc. Natl. Acad. Sci. U. S. A., 2004, 101, 17669-17674.

37 S. Manley and V. D. Gordon, Curr. Protoc. Cell Biol., 2008, 243, 1-13.

38 A. Moscho, O. Orwar, D. T. Chiu, B. P. Modi and R. N. Zare, Proc. Natl. Acad. Sci. U. S. A., 1996, 93, 11443-11447.

39 C. A. Schneider, W. S. Rasband and K. W. Eliceiri, Nat. Methods, 2012, 9, 671-675.

40 J. Mutterer and E. Zinck, J. Microsc., 2013, 252, 89-91.

41 P. J. Quinn, Prog. Lipid Res., 2010, 49, 390-406.

42 L. A. Bagatolli, Biochim. Biophys. Acta, 2006, 1758, 1541-1556.

43 E. Sezgin, I. Levental, M. Grzybek, G. Schwarzmann, V. Mueller, A. Honigmann, V. N. Belov, C. Eggeling, U. Coskun, K. Simons and P. Schwille, Biochim. Biophys. Acta, 2012, 1818, 1777-1784.

44 J. Juhasz, F. J. Sharom and J. H. Davis, Biochim. Biophys. Acta, 2009, 1788, 2541-2552.

45 S. Chiantia, J. Ries, N. Kahya and P. Schwille, ChemPhysChem, 2006, 7, 2409-2418.

46 S. L. Veatch and S. L. Keller, Phys. Rev. Lett., 2002, 89, 268101/ 1-268101/4.

47 S. L. Veatch and S. L. Keller, Phys. Rev. Lett., 2005, 94, 148101/ 1-148101/4.

48 N. Ahmad, R. Muhammad, H. A. Tajuddin and M. Misran, Colloids Surf., A, 2014, 443, 96-101.

49 K. Trajkovic, C. Hsu, S. Chiantia, L. Rajendran, D. Wenzel, F. Wieland, P. Schwille, B. Brügger and M. Simons, Science, 2008, 319, 1244-1247.

50 L. Yang, L. Ding and H. W. Huang, Biochemistry, 2003, 42, 6631-6635.

51 S. J. Marrink and A. E. Mark, Biophys. J., 2004, 87, 3894-3900.

52 T. Baumgart, G. Hunt, E. R. Farkas, W. W. Webb and G. W. Feigenson, Biochim. Biophys. Acta, 2007, 1768, 2182-2194.

53 O. G. Mouritsen, Eur. J. Lipid Sci. Technol., 2011, 113, 11741187.

54 N. I. Zahid, O. K. Abou-Zied, R. Hashim and T. Heidelberg, J. Phys. Chem. C, 2011, 115, 19805-19810.

55 N. I. Zahid, O. K. Abou-Zied, R. Hashim and T. Heidelberg, Langmuir, 2012, 28, 4989-4995. 